\title{
Therapeutic Drug Monitoring in Non-Tuberculosis Mycobacteria Infections
}

\author{
Jan-Willem Alffenaar ${ }^{1,2,3,4}$ - Anne-Grete Märtson ${ }^{4} \cdot$ Scott K. Heysell ${ }^{5} \cdot$ Jin-Gun Cho ${ }^{6,7,8} \cdot$ Asad Patanwala ${ }^{1,9}$. \\ Gina Burch $^{10}$ • Hannah Y. Kim ${ }^{1,2,3}$ - Marieke G. G. Sturkenboom ${ }^{4}$. Anthony Byrne ${ }^{11}$ - Debbie Marriott ${ }^{12}$. \\ Indy Sandaradura ${ }^{6,13} \cdot$ Simon Tiberi ${ }^{14,15} \cdot$ Vitali Sintchencko $^{3,16,17,18}$. Shashikant Srivastava ${ }^{19,20}$. \\ Charles A. Peloquin ${ }^{10}$
}

Accepted: 6 February 2021 / Published online: 10 March 2021

(c) The Author(s) 2021

\begin{abstract}
Nontuberculous mycobacteria can cause minimally symptomatic self-limiting infections to progressive and life-threatening disease of multiple organs. Several factors such as increased testing and prevalence have made this an emerging infectious disease. Multiple guidelines have been published to guide therapy, which remains difficult owing to the complexity of therapy, the potential for acquired resistance, the toxicity of treatment, and a high treatment failure rate. Given the long duration of therapy, complex multi-drug treatment regimens, and the risk of drug toxicity, therapeutic drug monitoring is an excellent method to optimize treatment. However, currently, there is little available guidance on therapeutic drug monitoring for this condition. The aim of this review is to provide information on the pharmacokinetic/pharmacodynamic targets for individual drugs used in the treatment of nontuberculous mycobacteria disease. Lacking data from randomized controlled
\end{abstract}

Jan-Willem C. Alffenaar and A. G. Märtson contributed equally to the article.

Jan-Willem Alffenaar

johannes.alffenaar@sydney.edu.au

1 School of Pharmacy, Faculty of Medicine and Health, University of Sydney, Pharmacy Building (A15), Sydney, NSW 2006, Australia

2 Westmead Hospital, Westmead, NSW, Australia

3 Marie Bashir Institute for Infectious Diseases and Biosecurity, University of Sydney, Sydney, NSW, Australia

4 Department of Clinical Pharmacy and Pharmacology, University Medical Center Groningen, University of Groningen, Groningen, The Netherlands

5 Division of Infectious Diseases and International Health, University of Virginia, Charlottesville, VA, USA

6 Westmead Medical School, Faculty of Medicine and Health, The University of Sydney, Sydney, NSW, Australia

7 Respiratory and Sleep Medicine, Westmead Hospital, Westmead, NSW, Australia

8 Parramatta Chest Clinic, Parramatta, NSW, Australia

9 Royal Prince Alfred Hospital, Sydney, NSW, Australia

10 Department of Pharmacotherapy and Translational Research, College of Pharmacy, University of Florida, Gainesville, FL, USA
11 St. Vincent's Hospital Sydney, Heart Lung Clinic, Sydney, NSW, Australia

12 Department of Microbiology and Infectious Diseases, St. Vincent's Hospital, Sydney, NSW, Australia

13 Centre for Infectious Diseases and Microbiology Laboratory Services, NSW Health Pathology, Westmead Hospital, Sydney, NSW, Australia

14 Division of Infection, Barts Health NHS Trust, Royal London Hospital, London, UK

15 Centre for Primary Care and Public Health, Blizard Institute, Barts and The London School of Medicine and Dentistry, London, UK

16 NSW Mycobacterium Reference Laboratory, Centre for Infectious Diseases and Microbiology Laboratory Services, NSW Health Pathology, Westmead Hospital, Wentworthville, NSW, Australia

17 Centre for Infectious Diseases and Microbiology, Westmead Institute for Medical Research, Westmead, NSW, Australia

18 Centre for Infectious Diseases and Microbiology-Public Health, Westmead Hospital, Westmead, NSW, Australia

19 Department of Immunology, UT Southwestern Medical Center, Dallas, TX, USA

20 Department of Pulmonary Immunology, UT Health Science Center at Tyler, Tyler, TX, USA 
trials, in vitro, in vivo, and clinical data were aggregated to facilitate recommendations for therapeutic drug monitoring to improve efficacy and reduce toxicity.

\section{Key Points}

There is an urgent need to optimize non-tuberculous mycobacterial treatment because of the potential for acquired resistance and toxicity, resulting in a high treatment failure rate.

In vitro, in vivo, and clinical data were aggregated to draft recommendations for therapeutic drug monitoring to improve efficacy and reduce toxicity.

A significant knowledge gap was noted, as currently used dosages have not been established based on pharmacokinetic/pharmacodynamic principles. In vitro hollow fiber infection model studies followed by clinical trials in nontuberculous mycobacteria should be conducted and accompanied with concurrent therapeutic drug monitoring studies to optimize treatment.

\section{Introduction}

Nontuberculous mycobacteria (NTM) are opportunistic pathogens that can cause a wide range of diseases from minimally symptomatic self-limiting infections to progressive and life-threatening disease of the respiratory system, central nervous system, lymph nodes, joints, skin, or the whole body (disseminated disease) [1]. The prevalence of NTM is increasing in regions where reporting infection is required or where surveillance studies have been performed with rates as high as 10 per 100,000 population in Australia and North America and 2 per 100,000 in Europe [2]. Patients with pre-existing lung diseases including asthma, chronic obstructive pulmonary disease, cystic fibrosis, and bronchiectasis or with immunodeficiencies (inherited or acquired) are more susceptible to NTM disease [1, 3, 4]. Disseminated NTM disease mostly manifests in patients who are immunocompromised. Nontuberculous mycobacteria is more common in people aged 50 years and older [2]. Additionally, pulmonary NTM disease can occur in people with anatomic abnormalities in the thoracic cage [5]. Because of the combination of increased prevalence, greater clinical awareness, more testing in resource-rich settings, and molecular diagnostics, more patients with
NTM infections are being recognized. To diagnose NTM disease, clinical signs, and symptoms, positive cultures from sputum or bronchial lavage or other sites, and exclusion of other diseases are necessary $[3,6]$.

Microbiologically, NTM can be subdivided into slow- and rapid-growing species. Mycobacterium avium complex (MAC), M. intracellulare, M. chimaera, $M$. kansasii, M. malmoense, and $M$. xenopi are the most frequently observed slow-growing species while the rapidgrowing species include $M$. abscessus complex and $M$. fortuitum complex [7]. It is important to remember that specific cultures must be requested for the isolation of mycobacteria.

In patients without clinical symptoms, it may be difficult to decide whether to start treatment as the natural history of untreated infection is not well understood. An alternative strategy of observation may be appropriate in certain circumstances [4]. Where treatment is required, the American Thoracic Society (ATS), the British Thoracic Society, and the ATS/European Respiratory Society/European Society of Clinical Microbiology and Infectious Diseases/Infectious Diseases Society of America provide guidance on the treatment options [3, $4,6]$. However treatment of NTM is challenging because drug susceptibility is highly variable, some clinical presentations do not immediately necessitate treatment, multiple drugs have to be combined into a regimen, prolonged duration of therapy is recommended (12 months post-sterilization of sputum cultures), and treatment is often not well tolerated because of drug toxicity [6]. Based on current poor treatment outcomes and the need for multiple drugs for a prolonged period, there is an urgent need to optimize treatment. In addition to the requirement for new microbiologically active and well-tolerated drugs, TDM has been recommended for aminoglycosides and in patients with presumed malabsorption and/or drug-drug interactions $[3,4,8]$. However, little information or practical guidance is available [4]. The aim of this review is to provide information on the pharmacokinetic/pharmacodynamic (PK/PD) targets for individual drugs used in the treatment of NTM disease as well as to identify knowledge gaps. Because of a lack of data from randomized controlled trials, in vitro, in vivo, and clinical data will be aggregated to facilitate recommendations for TDM to improve efficacy and reduce toxicity.

\section{Susceptibility Testing for NTM}

When making TDM-based decisions, it is important to include the susceptibility (minimum inhibitory concentration [MIC]) of the pathogen in addition to the drug exposure as the MIC is included in all PK/PD parameters (area 
under the concentration-time curve over MIC [AUC/ MIC], maximum concentration over MIC $\left[C_{\max /} \mathrm{MIC}\right]$, and time above MIC [ $T>\mathrm{MIC}])$. However, accuracy and assay variation must be accounted for when using MICs reported by clinical laboratories [9]. In contrast with $M$. tuberculosis, the correlation between culture-based drugsusceptibility test (DST) results for NTM and treatment outcomes is highly variable depending on the NTM species and antimycobacterial agent. The Clinical and Laboratory Standards Institute (CLSI) recommends clarithromycin and amikacin susceptibility testing only for MAC, clarithromycin and rifampicin for $M$. kansasii, and clarithromycin for $M$. abscessus complex [10] as in vitro resistance to these agents has been associated with poor clinical outcomes. A DST in cases of infections caused by rapidly growing mycobacteria (e.g., M. fortuitum complex) has also been recommended [10]. Interpretation of M. xenopi, M. malmoense, and $M$. simiae complex DST results should be made with caution because of the lack of clinical correlations. Inferences about potential resistance can be made from the species of NTM. For example, imipenem and cefoxitin are usually more active against $M$. abscessus complex while $M$. chelonae complex organisms are more susceptible to tobramycin [11]. The $M$. abscessus subspecies abscessus is often more drug resistant than other rapidly growing mycobacteria.

Phenotypic susceptibility testing can be performed using the broth microdilution method according to CLSI recommendations or disc diffusion techniques [10]. However, the CLSI method provides more robust breakpoints, standardization, and reproducibility. Agreement between the two methods is less reliable for some drugs such as cefoxitin and amikacin and some of the breakpoint concentrations for detection of clinically relevant resistance in NTM remain controversial [11]. The MAC breakpoints for amikacin have been recently revised to include separate values for intravenous and the newer liposomal formulation of amikacin [12].

Phenotypic testing for macrolides should include the extension of the incubation time of the organism in a macrolide-containing medium to 14 days to detect inducible resistance. Such resistance is enabled by the expression of an erythromycin ribosomal methyltransferase gene (or erm gene), which has been observed in many rapidly growing mycobacteria. Testing of slow-growing mycobacteria may require incubation for as long as 6 weeks. However, drug stability may influence MIC measurements as, with the exception of amikacin, all antimycobacterial drugs can degrade after 2 weeks of incubation in the media for a DST [13]. More recently, technical improvements in matrix-assisted laser desorption ionization-time of flight mass spectrometry has opened a possibility of a matrix-assisted laser desorption ionization-based DST using the matrix-assisted laser desorption ionization Biotyper antibiotic susceptibility test rapid assay [14].
Drug resistance in mycobacteria can be intrinsic and/or acquired. Many rapidly growing mycobacteria carry genes encoding proteins that methylate ribosomal DNA in a critical region of the large subunit (50S) ribosome. These genes confer in vitro resistance to macrolides. For example, M. abscessus spp. abscessus is usually resistant to clarithromycin because of the presence of the functional erm gene in contrast to $M$. abscessus ssp. massiliense, which possesses a non-functional erm gene and remains susceptible to clarithromycin. Mycobacterium abscessus is naturally susceptible to amikacin, variably susceptible to cefoxitin and imipenem, and resistant to most other antimicrobial drugs [15]. The testing of large numbers of NTM isolates indicated that they can acquire resistance to macrolides, quinolones, aminoglycosides, and other drugs [16]. Statistically significant differences were revealed in susceptibility/resistance between $M$. avium and $M$. intracellulare with $M$. avium strains at the species level resistant to higher concentrations of amikacin, clarithromycin, linezolid, and streptomycin. The isolates of $M$. avium were also significantly more resistant than $M$. kansasii to amikacin, ciprofloxacin, clarithromycin, doxycycline, ethambutol, ethionamide, isoniazid, linezolid, moxifloxacin, rifabutin, rifampicin, streptomycin, and trimethoprim/sulfamethoxazole [17]. There is an apparent in vitro synergy between amikacin and clofazimine leading to several fold decreases in MICs to both drugs in both rapidly and slowly growing mycobacteria [18]. Some new agents such as tedizolid and omadacycline have demonstrated in vitro potency against NTM [19].

Molecular mechanisms of intrinsic and acquired resistance against macrolides, aminoglycosides, linezolid, clofazimine, and bedaquiline have recently been elucidated [20]. These advances enabled molecular approaches to a DST. For example, the NTM-DR kit (Hain Lifescience $\mathrm{GmbH}$, Nehren, Germany) can detect intrinsic and acquired resistance in NTM to macrolides and aminoglycosides by identifying resistance-conferring mutations in erm [21], rrl, and $r r s$ genes. Importantly, these molecular approaches can also detect heteroresistance, i.e., the presence of a resistant subpopulation in otherwise phenotypically drug-susceptible mycobacteria [22]. The whole genome sequencing of clinical isolates can assist in DST inferences and offers the ultimate high-resolution approach for resistome (all the present resistant genes) prediction. The use of a DST can be justified for the detection of acquired resistance in patients with recurrent or non-resolving NTM infection or a history of previous treatment with macrolides or aminoglycosides. Patients with documented low drug exposure are at risk of acquired drug resistance. Therapeutic drug monitoring can play an important role in the identification of patients with low drug exposure, e.g., as a result of malabsorption or drug-drug interactions $[3,4,8]$. The TDM-derived exposure relative to the MIC is therefore used to prevent underdosing and mitigate the risk of acquired drug resistance [9]. 

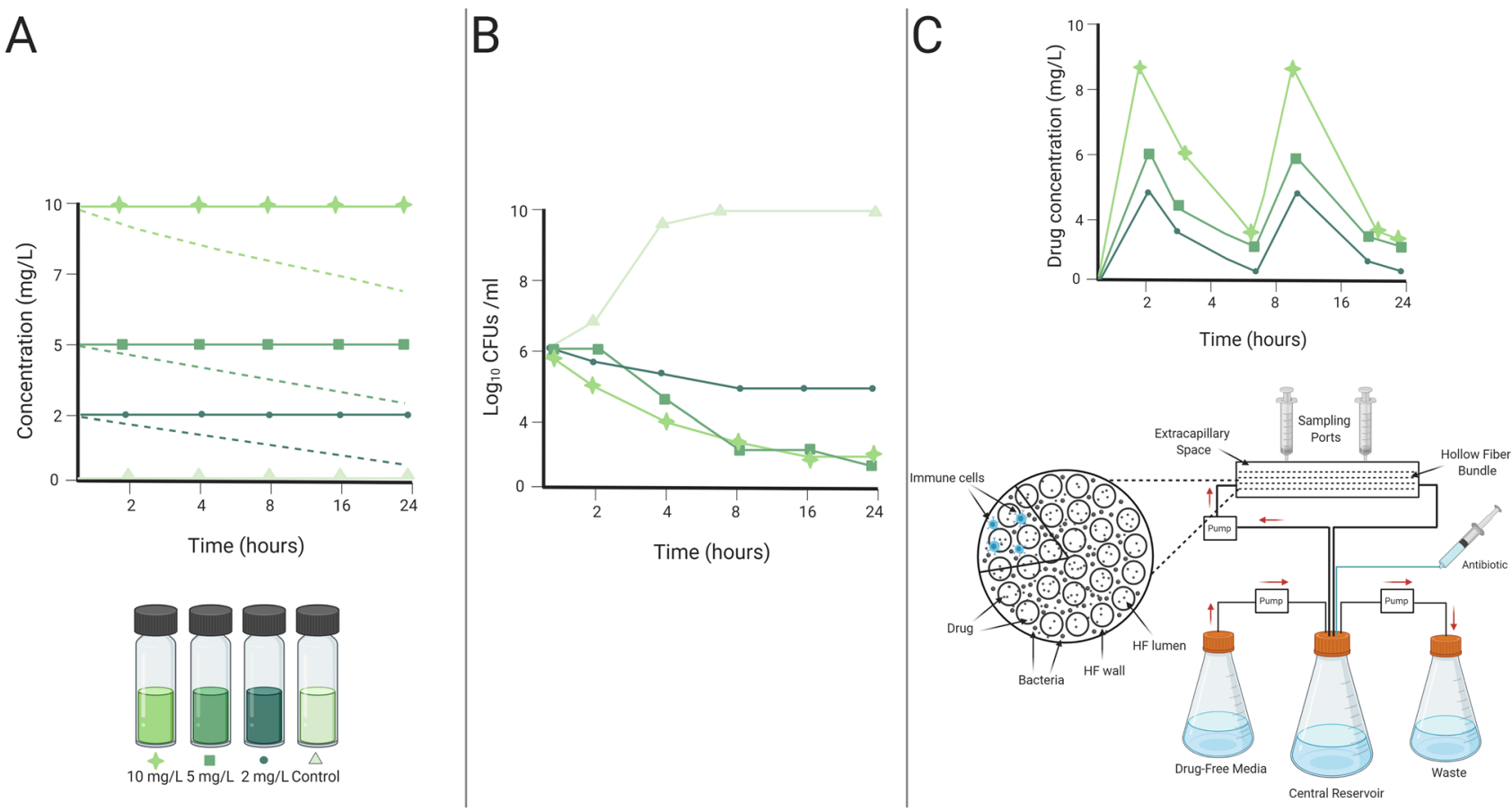

Fig. 1 In vitro infection models (created with BioRender.com). a Time-kill kinetic models study the bactericidal effect of static drug concentrations in time. The dashed line shows the decline in concentration as a function of chemical degradation due to instability of the compound. $\mathbf{b}$ The response of in vitro models is reflected by the number of colony-forming units (CFUs). An increase is observed for inactive compounds and a control situation (growth in medium). c Hol-

\section{Optimal Drug Exposures for the Treatment of NTM as Identified in the Hollow Fiber Infection Model}

Treatment success of any combination regimen largely depends upon the drug exposure achieved with the given drug dose in relation to the susceptibility of the pathogen. Suboptimal drug exposure results in the emergence of drug resistance [23]. Therefore, it is imperative to optimize the drug regimens using the $\mathrm{PK} / \mathrm{PD}$ principles described in preclinical models [24]. Time-kill kinetic in vitro experiments are straightforward and affordable to generate basic PD data [25] on drugs that are tested for NTM activity (Fig. 1a) [26, 27]. They can help to determine whether a drug has concentration- or time-dependent bactericidal activities. However, time-kill kinetic experiments use static drug concentrations over time while drug concentrations in humans are dynamic. To overcome this limitation, hollow fiber infection models have been developed using dynamic concentrations to better resemble human pharmacokinetics. In recent years, the hollow fiber infection model has been used to determine the optimal drug exposure for maximal kill and to develop combination regimens for NTM, namely MAC, M. kansasii, and M. abscessus [19, 20, 22-31] (Fig. 1b). This model offers low fiber infection models study the bactericidal effect of dynamic drug concentrations (mimicking human pharmacokinetics) in time. The hollow fiber infection model (HF) can facilitate experiments with extracellular bacteria, intracellular bacteria, and immune cells, thereby accounting for the different conditions of nontuberculous mycobacteria infections

the advantage of measuring the drug concentration changes with time (pharmacokinetics) as well as linking the shape of the concentration-time curve achieved in the systems with the antimicrobial effect of the drug (pharmacodynamics) [32]. The drug concentrations measured in the central compartment of the hollow fiber infection model represent the plasma concentration in patients. The subcellular compartment represents intracellular concentrations (as in cases of MAC and M. kansasii), which provide information on the drug penetration and accumulation of drug in the compartment. Finally, drug concentrations measured inside the bacteria are used to study the mechanism of drug action. However, a model to better mimic the complex human immune system where different immune cells are mixed and then exposed to bacteria followed by drug treatment could better decipher the role of the immune system in disease containment and treatment success (Fig. 1c).

The efficacy of the treatment regimen of azithromycin and ethambutol was tested in the MAC hollow fiber infection model and demonstrated a maximal kill of 1.52 $\pm 0.43 \log _{10}$ colony-forming units $(\mathrm{CFU}) / \mathrm{mL}$ during the first 7 days after which the regimen failed [33]. However, the addition of rifabutin has shown to keep the bacterial burden below stasis until the end of the study (day 28 , 


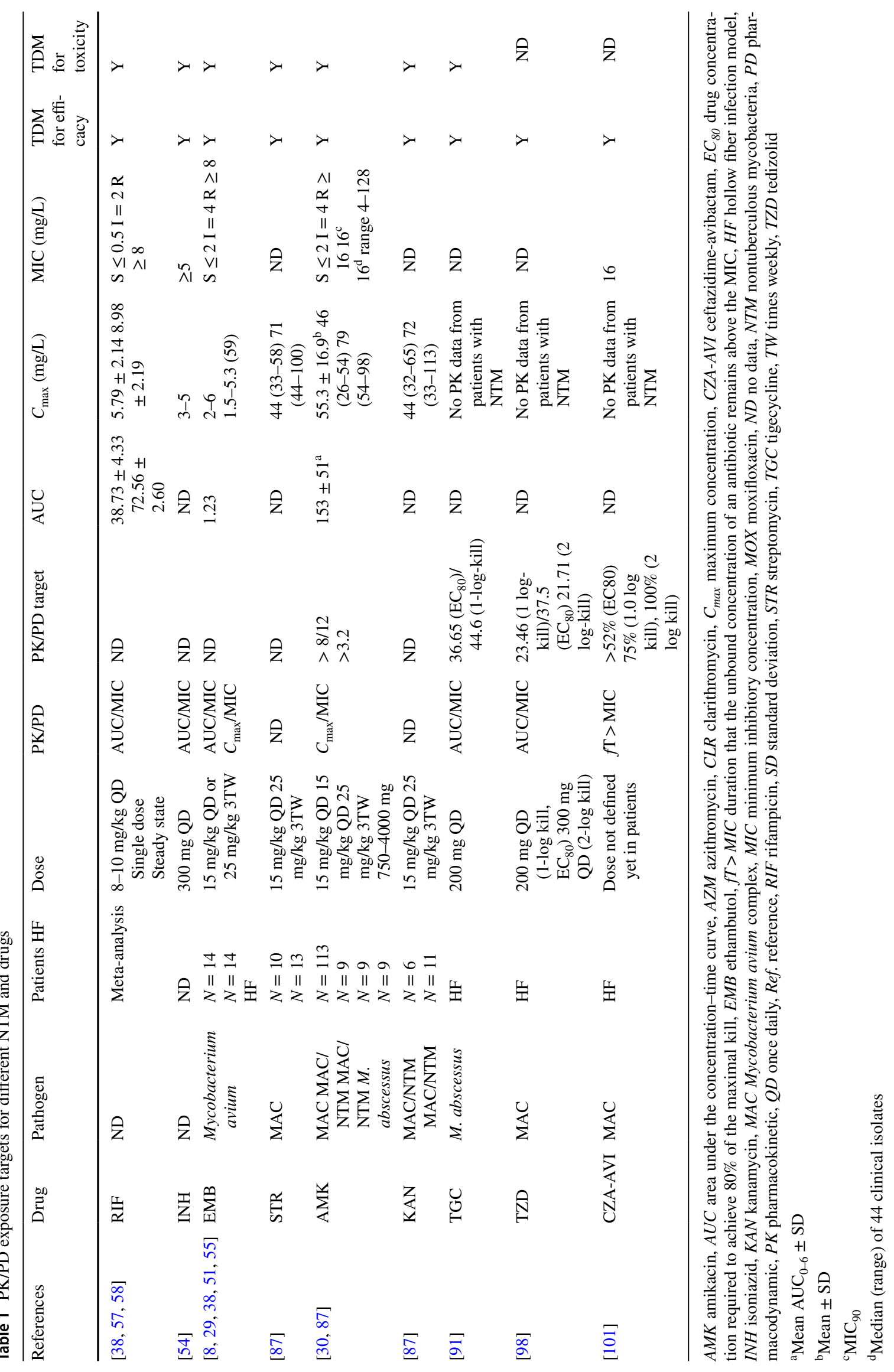


unpublished data). Currently, a randomized controlled trial evaluating rifampicin \pm a backbone of azithromycin/ethambutol is ongoing [34]. Thus, the macrolide-containing regimen needs the addition of rifamycin for a successful treatment outcome. Similarly, the M. kansasii hollow fiber infection model was used to compare the treatment regimens recommended by the ATS consisting of isoniazid, rifampicin, and ethambutol with the regimen recommended by the British Thoracic Society consisting of a combination of rifampicin, ethambutol, and clarithromycin [35]. The kill below stasis with the British Thoracic Society regimen was $3.07 \log _{10}$ CFU/mL compared with the $1.72 \log _{10}$ CFU/mL with the ATS regimen. Furthermore, while the British Thoracic Society regimen continued to kill $M$. kansasii during the 28-day study period, the ATS regimen failed because of the emergence of drug resistance [36].

Optimal PK/PD exposure targets, derived from the hollow fiber infection model, can form a basis for TDM. It must be emphasized that adequate follow-up is essential after dose adjustment [21]. The PK/PD targets from the hollow fiber infection model are summarized in Table 1.

\section{Use of TDM to Assess Clinical Response}

In the absence of a randomized controlled trial of TDM for a specific drug or regimen for a NTM species, there is an urgent need to replicate pre-clinical models of species-specific pharmacokinetics/pharmacodynamics and predict treatment outcomes. Pending the results of such trials, clinicians with access to TDM may prioritize use for specific patients at a higher risk of malabsorption, drug-drug interactions, or altered drug clearance, such as people living with human immunodeficiency virus, cystic fibrosis, diabetes mellitus, and obesity [37]. For example, concomitant use of rifampicin can lower clarithromycin and azithromycin total serum exposure AUC by $73 \%$ and 24\%, respectively [38]. Linezolid concentrations are also known to be decreased by rifampicin and increased by clarithromycin $[39,40]$. When measuring drug concentrations, it is important to take into account the anatomic locations of the infection such as bone and joint infection and antibiotic penetration into these regions as concentrations at the site of infection may be lower or higher than measured in plasma. Macrolides such as clarithromycin and azithromycin have respiratory epithelial fluid and macrophage concentrations estimated at 2-100 times and 400-800 times, respectively, in excess of their plasma concentrations [41]. Moreover, TDM can be utilized in cases where prolonged treatment durations are planned for agents where the risk of toxicity can be reduced with lower total exposures, such as ototoxicity and nephrotoxicity of aminoglycosides or the neurotoxicities and myelosuppression of the oxazolidinones [42, 43]. Taken together, the proportion of patients with NTM infections that may potentially benefit from TDM represents the majority of those commenced on therapy.

Nevertheless, logistical and perceived barriers to TDM implementation exist. Where TDM has been more routinely implemented, for instance, in intensive care medicine [44], educating medical professionals about the rationale and potential benefits, creating a standardized protocol and set of recommendations for sample collection, and dose adjustment and utilization of the same experienced referral laboratory have facilitated uptake [45, 46]. Given the inter-individual PK variability, repetition of TDM following dose adjustment or a change in interacting drugs is recommended. Additionally, while the most important PK/PD parameter for many antimycobacterial drugs is the AUC relative to the MIC, there can be some difficulties implementing these tests. For example, MIC testing of the infecting mycobacterial isolate may not be performed by some clinical laboratories and the interpretation of MIC results is not standardized for most drugs and NTM species, as well as certain genetic mutations (as discussed previously) may be a more important determinant than phenotypic susceptibility. Furthermore, because of the practical constraints of phlebotomy, few samples in the dosing interval are used (limited sampling strategies) [47, 48], as an approximation of AUC. Importantly, patient-centered expectations of treatment response, such as microbiological cure vs toxicity-free symptom improvement must be agreed upon, ideally prior to treatment initiation, and then re-assessed at an appropriate interval following TDM. A typical case of MAC is described in Text box 1 and the application of TDM is presented in Text box 2 .

Text box 1 Case report

\footnotetext{
The first case is a 15-year-old man with cystic fibrosis who weighed $66 \mathrm{~kg}$ (body mass index of $20.8 \mathrm{~kg} / \mathrm{m}^{2}$ ), had persistently positive sputum cultures for Mycobacterium avium complex (MAC) [not further speciated] with retained macrolide susceptibility, nodular bronchiectatic lung disease, and worsening lung function. Treatment for MAC was initiated with the goal of symptom and lung function improvement and a decrease in MAC burden. Initially, he was placed on azithromycin at $500 \mathrm{mg}$ daily, rifampicin $600 \mathrm{mg}$ daily, and ethambutol $800 \mathrm{mg}$ daily. Therapeutic drug monitoring was performed with a limited sampling strategy and estimated maximum concentration of azithromycin was $0.16 \mathrm{mg} / \mathrm{L}$ (expected range $0.20-0.70 \mathrm{mg} / \mathrm{L}$ ) and rifampicin was $3.02 \mathrm{mg} / \mathrm{L}$ (expected range $8-24 \mathrm{mg} / \mathrm{L}$ ). Azithromycin was increased to $750 \mathrm{mg}$ daily and rifampicin to $900 \mathrm{mg}$ daily with subsequent tolerance and decrease of sputum mycobacterial burden. Following addition of clofazimine and inhaled amikacin, 1 year of sustained MAC clearance was achieved.
} 
Text box 2 Recommendations for TDM in NTM disease

\begin{tabular}{|c|c|}
\hline Efficacy & $\begin{array}{l}\text { Information on PK/PD targets on drugs used for NTM is scarce. TDM for efficacy is preferably guided by } \\
\text { PK/PD targets but if these are lacking it would help to target normal exposure in patients with low expo- } \\
\text { sure not responding to therapy }\end{array}$ \\
\hline Toxicity & $\begin{array}{l}\text { In the case of presumed drug-related toxicity, dose adjustment is suggested when high drug concentrations } \\
\text { are observed at a standard dose }\end{array}$ \\
\hline Drug-drug interactions & $\begin{array}{l}\text { Drug-drug interactions can be managed as usual and TDM can be used to verify if exposure is within the } \\
\text { normal range for that particular drug }\end{array}$ \\
\hline Renal or hepatic function problems & $\begin{array}{l}\text { For renally excreted or hepatically metabolized drugs, dosages can be adjusted as recommended by guide- } \\
\text { lines and TDM can be used to verify if exposure is within the normal range for that particular drug }\end{array}$ \\
\hline $\begin{array}{l}\text { Comorbidities (e.g., diabetes mel- } \\
\text { litus, HIV, CF) }\end{array}$ & $\begin{array}{l}\text { As treatment in patients with comorbidities is often accompanied with complications, it is recommended to } \\
\text { evaluate drug exposure in situations where these may arise }\end{array}$ \\
\hline Sampling & $\begin{array}{l}\text { At least } 2 \text { samples should be collected during the dose interval to assess drug exposure. A single sample } \\
\text { often does not provide sufficient information as delayed absorption on reduced clearance is imperceptible }\end{array}$ \\
\hline
\end{tabular}

$C F$ cystic fibrosis, $H I V$ human immunodeficiency virus, $N T M$ nontuberculous mycobacteria, $P D$ pharmacodynamic, $P K$ pharmacokinetic, $T D M$ therapeutic drug monitoring

\section{Drugs}

A non-systematic literature search was performed in PubMed using the keywords pharmacokinetics, pharmacodynamics, NTM, nontuberculous mycobacteria, and the various drugs as included in the treatment guidelines $[4$, 6]. Table 1 summarizes the optimal PK/PD exposure targets for drugs, as monotherapy. In the hollow fiber infection model, the optimal exposure is defined as the drug concentration required to achieve $80 \%$ of the maximal kill, it can be expressed as the PK/PD target for that particular drug, e.g., as an AUC/MIC ratio. Based on the AUC/MIC target ratio, drug exposure at difference dosages and the susceptibility breakpoint, it can be estimated which percentage of patients can be successfully treated with that drug. This strategy has been used earlier to translate in vitro PK/PD results to clinical practice [49]. The next section includes an overview of in vitro and in vivo studies that have shown efficacy or inefficacy when used for NTM. In addition, potential PK/PD indices and targets are discussed to guide therapy. In cases where no or limited data are available, we identified this as a knowledge gap.

\subsection{Rifamycins}

Rifampicin, rifabutin, and rifapentine are bactericidal drugs that inhibit bacterial DNA-dependent RNA-polymerase to inhibit RNA synthesis [50]. Rifampicin and rifabutin have a wide range of activity against NTM including MAC, $M$. malmoense, $M$. xenopi, and rifamycin-sensitive $M$. kansasii [51]. Although M. abscessus has been known to be resistant to rifamycins, rifabutin has recently shown activity against M. abscessus [52], demonstrating the future potential of rifamycins as repurposed drugs [53].
Because of a poor correlation between in vitro MIC data for rifampicin and rifabutin and MAC, a DST is not performed for rifamycins, except for M. kansasii in which resistance data may help determine the choice of antibiotic regimen [54]. Rifamycins alter the concentration of macrolide antibiotics $[38,55,56]$ but are themselves not significantly affected by concurrent treatment with macrolides [38]. Although there are no data on PK/PD targets specifically for nontuberculous mycobacteria, at current recommended doses only $18 \%$ of patients taking rifampicin $(n=$ 299) for pulmonary MAC infection met the PK/PD target associated with a bactericidal effect against $M$. tuberculosis in the hollow fiber model, and only $6 \%$ had total AUC 0-6/MIC bactericidal ratios against $M$. tuberculosis in the mouse model; however, the $\mathrm{AUC}_{0-6}$ were compared to AUC 0-24 index values from the published literature [38]. Therefore, much higher doses of rifampicin may be required to achieve PK/PD targets [57]. If higher doses are considered, TDM may be beneficial in minimizing the risk of rifampicininduced toxicity [58].

\subsection{Macrolides}

The macrolide antibiotics indicated for NTM include azithromycin and clarithromycin. These antibiotics have an effect through inhibiting protein synthesis with binding to the bacterial 50S ribosomal subunit [59]. Both have a similar spectrum of activity that includes MAC, M. kansasii, $M$. malmoense, and M. abscessus [38, 60-62].

Regarding M. abscessus complex, the kill below stasis with the combination regimen of amikacin, cefoxitin, and clarithromycin was $1.22 \log _{10} \mathrm{CFU} / \mathrm{mL}$ and in the hollow fiber infection model the therapy failed after 14 days of the treatment [36]. Azithromycin and clarithromycin are extensively distributed into tissues and macrophages. Tissue concentrations may be up to 30- and 100-fold higher 
than serum concentrations for clarithromycin and azithromycin, respectively $[63,64]$. In patients with MAC, the peak serum concentrations of clarithromycin have been shown to be lower than the MIC required for susceptibility [38, 56]. Moreover, a study reported low $C_{\max }$ values in patients who had a $74 \%$ favorable microbiological response [56]. However, $C_{\max }$ might not be the best PK/PD target for macrolides, as AUC/MIC has been used previously in tuberculosis (TB) [65, 66]. Furthermore, in the described study, the patients were receiving intermittent and daily therapies and sub-target concentrations were found in the majority of the patients, which makes it difficult to discuss differences [56]. Currently, there is also no cut-off serum concentration value to assess toxicity. Thus, current evidence does not support the use of TDM for macrolide therapy, except to rule out malabsorption.

\subsection{Fluoroquinolones}

Fluoroquinolones occasionally play a role in NTM regimens, but their role has not been well defined. They exert their antibacterial effect by inhibiting DNA gyrase, which disrupts DNA synthesis. Fluoroquinolones exhibit concentrationdependent activity.

Ciprofloxacin and moxifloxacin have activity against $M$. avium complex and $M$. intracelluare, but MICs tend to be higher in M. intracellulare [67]. Fluoroquinolones also have some activity against other slow-growing mycobacterial species (M. xenopi, M. kansasii, M. szulgai). Ciprofloxacin may be synergistic with ethambutol against $M$. malmoense [68]. Data describing susceptibility for $M$. abscessus are conflicting with regard to fluoroquinolone activity $[69,70]$.

Hollow fiber infection model studies suggest standard moxifloxacin doses (400 $\mathrm{mg}$ daily) are inadequate for treating disseminated MAC [49, 71]. Moxifloxacin has been tested against $M$. abscessus strains, where regrowth was observed beyond 3 days and subsequent Monte Carlo simulations suggested that $400-800 \mathrm{mg} /$ day doses would achieve $80 \%$ of the maximal kill in $\leq 12.5 \%$ of simulated patients [49]. Moreover, in another hollow-fiber infection model of M. kansasii, it has been shown that moxifloxacin has a higher kill rate than the isoniazid, rifampicin, and ethambutol regimen [72]. Clinical PK studies on fluoroquinolones are lacking in NTM.

\subsection{Clofazimine}

The activity of clofazimine has been well established for $M$. leprae (leprosy) since the 1950s. A PK analysis of clofazimine in healthy subjects reported a delayed absorption (0-4 h), and up to a 2.5-fold increase in exposure when given with a high-fat meal [73]. Minimum inhibitory concentrations of clofazimine against NTM species are variable. A recent study reported clofazimine MICs for clinical isolates of MAC from 0.031 to $8 \mathrm{mg} / \mathrm{L}$, while for $M$. abscessus, MICs had a range of $0.031-16 \mathrm{mg} / \mathrm{L}$. All patients with MIC $\leq 0.25 \mathrm{mg} / \mathrm{L}$ achieved culture conversion, with this cut-off being associated with a significant likelihood for culture conversion compared to MIC $>0.5 \mathrm{mg} / \mathrm{L}$ (odds ratio 39.3 , $p=0.021$ ) [74]. Clofazimine has shown synergy with amikacin against $M$. abscessus, $M$. chelonae, $M$. fortuitum, and MAC $[18,75]$ and clinical guidelines support the use in $M$. abscessus [51]. A recent in vitro synergy study demonstrated that clofazimine extended the bacteriostatic activity of bedaquiline against $M$. abscessus although with increased bedaquiline resistance. Clofazimine showed a slight bactericidal activity against $M$. avium and slowed bedaquiline resistance for $M$. avium [76]. In a hollow fiber infection model, clofazimine has shown modest effect against $M$. kansasii [77]. Moreover, in an in vivo mice model, it was shown that inhaled clofazimine reaches four times higher concentrations in lung tissue compared with oral clofazimine [78]. Retrospective studies have suggested that clofazimine can effectively replace rifampicin in the MAC regimen containing ethambutol and a macrolide $[79,80]$ and has been suggested to be potentially an effective drug in combination with other antimicrobials against NTM [81]. In vitro synergy by the addition of clofazimine to other drugs would need to be evaluated in pre-clinical models such as a hollow fiber system to optimize regimens based on mimicked human kinetics and drug exposure. The role of TDM for clofazimine is unclear, it might be useful in instances with extremes of body fat deposition [82]. Future studies to establish dosing regimens for NTM could provide evidence for a dose-exposure-response relationship, target PK/PD markers, and thresholds required for TDM.

\subsection{Isoniazid}

Isoniazid has been used for MAC, M. kansasii, and $M$. xenopi [51]. Factors of PK variability include a high-fat meal (12\% decrease in AUC with a high-fat meal) and polymorphisms in $\mathrm{N}$-acetyltransferase 2, with the latter contributing to hepatotoxicity $[8,83]$. Isoniazid MICs for wild strains of $M$. kansasii range from 0.5 to $5.0 \mathrm{mg} / \mathrm{L}$ using agar or broth methods; however, breakpoints against NTM have not been established [54].

A prospective PK study in patients with NTM reported isoniazid $C_{\max }$ of 5.0 and $4.9 \mathrm{mg} / \mathrm{L}$ in two patients, in line with the normal observed range $(3-6 \mathrm{mg} / \mathrm{L})$ [51, 55]. In a hollow-fiber infection model, a standard regimen consisting of isoniazid, rifampicin, and ethambutol showed efficacy against $M$. kansasii with a low kill rate of $-0.08 \pm 0.05$ $\log _{10} \mathrm{CFU} / \mathrm{mL} / \mathrm{day}$, in contrast to moxifloxacin monotherapy demonstrating the highest kill rate of $-0.82 \pm 0.15 \log _{10}$ 
$\mathrm{CFU} / \mathrm{mL} /$ day [72]. A target $C_{\max }$ of 3-5 mg/L has been suggested in patients with NTM with malabsorption or poor response [51]. The AUC/MIC may provide a more robust TDM approach, but further PK/PD studies are required in patients with NTM to establish and validate the target ranges.

\subsection{Ethambutol}

Ethambutol, which inhibits the synthesis of the cell wall, is used for MAC, M. kansasii, M. malmoense, M. xenopi [51], and $M$. marinum. The CLSI-defined MIC resistance breakpoint ( $>4 \mathrm{mg} / \mathrm{L}$ ) is limited to $M$. kansasii and M. marinum [54], and in vitro susceptibility does not well predict clinical outcome, especially for MAC [75].

In a hollow-fiber model of intracellular $M$. avium, the microbial kill rate was associated with $C_{\max } / \mathrm{MIC}$, with a value of 1.23 in serum to achieve $90 \%$ of the maximal kill [29]. Monte Carlo simulations of 10,000 patients also suggested the higher ethambutol dose of $\geq 50 \mathrm{mg} / \mathrm{kg}$ twice a week rather than current doses of $15 \mathrm{mg} / \mathrm{kg} / \mathrm{day}$ to achieve a target attainment probability of above $80 \%$ for disseminated M. avium disease [29].

A prospective PK study in patients with NTM showed a $C_{\max }$ of ethambutol in NTM to be comparable to patients with TB (2-6 mg/L) (Table 1) [55]. Optic neuritis is believed to be a concentration-dependent toxicity [8]. Current recommendations include a target $C_{\max }$ of $2-6 \mathrm{mg} / \mathrm{L} \mathrm{[51]} \mathrm{and} \mathrm{a}$ case-by-case $C_{\max } / \mathrm{MIC}$ in patients with altered pharmacokinetics (e.g., cystic fibrosis, renal impairment), malabsorption, and poor response [84].

\subsection{Aminoglycosides}

Aminoglycosides act primarily via binding to the 30 S ribosomal subunit, leading to inhibition of the bacterial protein synthesis. They have a concentration-dependent effect, and toxicity is associated with cumulative drug exposure [85, 86]. Pharmacokinetic/pharmacodynamic data of aminoglycosides have largely been derived from other infections with Gram-negative bacteria [86]. Amikacin can be utilized for MAC and M. abscessus complex infections, while tobramycin is more active in vitro than amikacin for $M$. chelonae [85].

A hollow fiber infection model for M. abscessus was developed to mimic the pulmonary pharmacokinetics of amikacin [30]. Both efficacy and acquired drug resistance were related to $C_{\max } / \mathrm{MIC}$ ratios [30]. However, Monte Carlo simulations of 10,000 patients showed that a standard dose of $1500 \mathrm{mg}$ of amikacin will only achieve a $C_{\max } / \mathrm{MIC}$ ratio of 3.2 in $21 \%$ of patients. Doses of $4000 \mathrm{mg}$ of amikacin per day were needed to attain this ratio in $70 \%$ of the patients, but these doses carry unacceptably high risks of ototoxicity and nephrotoxicity.

Concerning safety, $25 \mathrm{mg} / \mathrm{kg}$ of aminoglycoside three times a week was compared with $15 \mathrm{mg} / \mathrm{kg}$ five times a week in both TB- and NTM-infected patients [87]. The size of the dosage and the frequency of administration were not associated with the incidences of ototoxicity, vestibular toxicity, or nephrotoxicity. Risk of ototoxicity was associated with increased age, longer duration of treatment, and larger cumulative dose received [87]. Therapeutic drug monitoring of aminoglycosides is primarily focused on prevention of toxicity.

In 2018, the US Food and Drug Administration approved amikacin lipid inhalation suspension (Arikayce ${ }^{\circledR}$ ) as part of a combination drug regimen in adult patients with complicated MAC lung disease. The recommended dose is 590 $\mathrm{mg} / 8.4 \mathrm{~mL}$ as a once-daily inhalation. Mean systemic exposure of amikacin after inhalation is low ( $C_{\max } 25$-fold and AUC 6-fold lower compared with $15 \mathrm{mg} / \mathrm{kg} /$ day of intravenous amikacin). Little to no accumulation occurred following multiple once-daily doses for 3 months. Ototoxicity was observed more frequently in the guideline-based therapy with inhaled amikacin (17.0\%) compared with the guidelinebased therapy $(9.8 \%)$. The difference was primarily driven by the occurrence of tinnitus and dizziness. Nephrotoxicity was observed infrequently in clinical trials investigating inhaled amikacin, occurring in a similar proportion as control groups [88].

\subsection{Tetracyclines}

For rapidly growing NTM such as M. fortuitum, M. abcessus, M. chelonae, and M. smegmatis, in vitro studies demonstrate tigecycline exhibits excellent antimicrobial activity [89]. The MIC for tigecycline to each of these isolates was very low $(<1 \mathrm{mg} / \mathrm{L})$ with $100 \%$ susceptibility as compared with minocycline and doxycycline that showed high MICs ( $>16$ $\mathrm{mg} / \mathrm{L})$ and resistance to $>60 \%$ of $M$. abscessus and $M$. chelonae isolates [89]. The situation was somewhat reversed for the slow-growing NTM including M. kansasii and $M$. marinum where tigecycline performed poorly but $100 \%$ of isolates were susceptible to minocycline. Unfortunately, all tetracyclines exhibited poor antimicrobial activity against MAC [89]. Two specific ribosomal resistance protein genes, $\operatorname{otr}(A)$ and $\operatorname{tet}(M)$, have been identified among tetracyclineresistant mycobacteria [62]. Moreover, to achieve adequate concentrations in the lungs, tigecycline requires twice-daily dosing [90].

The AUC/MIC is likely to be the most useful PK/PD measure for tetracyclines that correlates with efficacy [91]. Therapeutic drug monitoring may be useful for the efficacy of doxycycline and minocycline when treating the slowgrowing NTM such as M. kansasii and may be useful for 
reducing tigecycline toxicity. The efficacy of a novel oral tetracycline in NTM disease, omadacycline, is currently unknown; however, it has shown a relatively good safety profile [92] and it has been used in NTM disease. In addition, it has shown to have efficacy against $M$. abscessus subspecies abscessus in an in vitro study [93]. However, recently, the marketing authorization approval was removed from the European Medicines Agency by the company for business reasons [94].

\subsection{Oxazolidinones}

There are limited data concerning linezolid and NTM but several studies highlight the importance of species identification as a guide to susceptibility. In vitro studies performed on isolates of the most common clinically significant NTM, isolates of the MAC, demonstrated that linezolid was highly bactericidal. Because of the MIC distribution, doses up to $1800 \mathrm{mg} /$ day were required to optimize the bactericidal effect, resulting in a high adverse event rate [95]. A recent publication testing antimicrobial susceptibilities of the newly recognized pathogen $M$. chimaera [96] demonstrated 39\% of isolates (34/87 isolates) had intermediate susceptibility and 39\% (34/87 isolates) were resistant to linezolid. Of 37 M. abscessus and $M$. fortuitum isolates tested by Zhang et al. [69], 15\% and 18\%, respectively, were resistant to linezolid.

Tedizolid may have significant advantages over linezolid although clinical data remain limited. The high oral bioavailability and a longer half-life of tedizolid $(11.0 \mathrm{~h}$ vs 5.0 h) enable once-daily dosing. However, the protein binding of $70-90 \%$ for tedizolid vs $30 \%$ for linezolid suggests that hypoalbuminemia in chronic disease may have an impact on drug concentrations. Most phase II-III trials studies have been performed over 6 days and long-term usage has not been well described. For treatment courses up to 7 days, tedizolid appears to be better tolerated than linezolid, especially in regard to hematological adverse events such as anemia and thrombocytopenia [97]. Hollow fiber infection models have shown the effect of tedizolid in NTM (Table 1) [35, 98]. Although there are limited data suggesting in vitro activity against MTB and NTM and it is being used in clinical practice, there is only a single case report of tedizolid use in M. avium infection following linezolid toxicity [95].

\subsection{Beta-Lactams}

Cefoxitin and imipenem are currently used for the treatment of rapidly growing NTM infections with organisms such as M. abscessus complex and M. chelonae [6, 51]. These betalactams are more resistant to hydrolysis by mycobacterial beta-lactamases, which may explain their observed clinical efficacy. The recent discovery of the potent inhibitory effect by avibactam against beta-lactamases in $M$. abscessus and $M$. avium has re-focused attention on beta-lactams for the treatment of NTM. Treatment with avibactam lowered MICs of M. abscessus and M. avium 4- to 32-fold for several betalactams [99-101].

The instability of beta-lactam compounds and the longer generation times of mycobacteria complicates drug susceptibility testing and the performance of PD experiments investigating beta-lactam/beta-lactamase combinations, which has resulted in a paucity of PK/PD data [102]. Ceftazidime/ avibactam has been shown to have an effect against MAC (Table 1) [101]. Mycobacterium avium complex eradication with ceftazidime/avibactam was achieved at $0.61-2.4 \log _{10}$ $\mathrm{CFU} / \mathrm{mL}$ below stasis [101]. Because of a lack of clinical studies, the optimal dosing regimen for the treatment of NTM infections is yet to be defined.

\subsection{Bedaquiline/Delamanid/Pretonamid}

Bedaquiline, delamanid, and pretonamid have been developed specifically for TB. However, only bedaquiline has been demonstrated to have some activity against NTM, while delamanid has very limited activity [103]. Notwithstanding the inhibition, bedaquiline appears to lack in vitro bactericidal activity against NTM species, showing only modest bacteriostatic activity [104]. Bedaquiline inhibits ATP-synthase and is bacteriostatic against $M$. avium, $M$. ulcerans, and M. abscessus [104-106]. It is bacteriostatic with the following rapid growers: $M$. abscessus and M. chelonae, and bactericidal for M. fortuitum. Rapid-growing $M$. xenopi is resistant to bedaquiline [107].

This seems to be the case in vivo as well with the efficacy of bedaquiline appearing to be limited. In mouse models, bedaquiline demonstrated bacteriostatic activity and reduced M. abscessus CFU by $1 \log$ [108], but no survival benefit was appreciated. A small case series of patients given bedaquiline with drug-resistant MAC or M. abscessus as a compassionate use treatment reported a microbiological response in six of ten patients, but only a few patients' sputum culture converted at 6 months [106], and relapse with bedaquiline-resistant strains has been reported [109].

Delamanid inhibits mycolic acid synthesis and possesses in vitro activity against $M$. kansasii and $M$. bovis, it is not active against $M$. avium, M. chelonae, M. abscessus, or M. fortuitum [110], though more studies are needed to understand activity against other NTM. Pretonamid, a novel nitrimidazole licensed to treat drug-resistant TB, has so far been shown to have little or no activity against NTM. 


\section{Discussion and Conclusions}

In theory, TDM for NTM makes perfectly good sense [4]. The NTM species are at least as resistant, if not more resistant, to available drugs when compared with M. tuberculosis. Pharmacokinetics/pharmacodynamics have progressed considerably for TB, and many experts routinely use TDM to manage TB, multi-drug resistant TB, and extensively drugresistant TB. By comparison, the treatment for NTM has not evolved to incorporate TDM.

Nevertheless, data are lacking for drug treatment choices, drug doses, and desirable concentrations for NTM infections. None of the currently used drugs was specifically developed for NTM, and the 'normal ranges' have been borrowed from other disease states such as TB as a placeholder for future developments. In some cases, the ranges have been holding their place since the 1990s, with no reprieve in sight.

The treatment of NTM largely has developed based on expert opinion and extrapolation, using available drugs at the 'usual' doses because of limited data. Further clinical trials including PK/PD analyses are urgently needed with a focus on shorter, better, and more tolerable treatment. Because many patients with pulmonary MAC and other NTM are older and more frail, drug intolerance is common. Even when faced with measured low plasma drug concentrations, physicians often are reluctant to increase the doses because of concerns of adverse effects.

What truly is needed are drugs designed for specific NTM. Actual pharmacokinetics/pharmacodynamics for each organism, beginning in vitro with a hollow fiber infection model, would allow for smarter drug therapy in patients with NTM. Moreover, clinical trials looking into specific knowledge gaps in NTM should be conducted and accompanied with concurrent TDM studies, where specific PK/PD targets and other necessary endpoints should be considered [111]. Drugs need to be substantially more tolerable than, for instance, tigecycline and clarithromycin. They need to be more potent for the varying metabolic states of NTM and the microenvironments such as biofilms where they often reside, as well as having a better balance between efficacy and toxicity. There are still many problems in developing new drugs for NTM as the disease itself, as mentioned before, may vary in clinical severity from few or no complaints to life-threatening disease. In addition, it has been recently discussed in a review that consideration should be given to whether the aim is to develop curative or maintenance treatment, in the context of the heterogeneous nature of NTM microbes and multiple other factors [112]. The observed increasing prevalence of NTM lung disease $[113,114]$ and the frequent life-long burden of disease amongst infected patients should make NTM disease and its treatment a research priority as clearly no effective and tolerable short-course treatment is available. The success of recent public private partnerships to catalyze drug development for TB may serve as a model for the NTM research community [115].

In summary, the application of TDM to optimize NTM treatment could be useful, maximizing efficacy while minimizing toxicity. As we have shown, data supporting PK/PD targeted dosing are scarce. In the absence of such data, we advocate for targeting expected drug exposures in patients with documented low exposure who are not responding to therapy. The same applies for toxicity, as it is advisable to reduce the dose in a patient with drug concentration-associated toxicity. Therapeutic drug monitoring can be of further help in the management of drug-drug interactions, and reduced drug clearance in the case of renal function loss. Therapeutic drug monitoring may serve as a critical bridge to new drugs and drug regimens that are critically needed for durable NTM cure without treatment-related morbidity. As mentioned, more research is needed to optimize treatment and for TDM to support such treatment, but currently we are a long way from reaching it.

\section{Declarations}

Funding No sources of funding were received for the preparation of this article.

Conflict of interest Anne-Grete Märtson was funded by Marie Skłodowska-Curie Actions (grant agreement no. 713660-PRONKJEWAIL-H2020-MSCA-COFUND-2015). Scott K Heysell was supported by NIH R01 AI137080. Shashikant Srivastava received supported by the ATS Foundation/Insmed Research Award in Non-Tuberculous Mycobacteria Lung Disease and NIH 1 R21 AI148096-01. Jan-Willem Alffenaar, Jin-Gun Cho, Asad Patanwalla, Gina Burch, Hannah Y Kim, Marieke GG Sturkenboom, Anthony Byrne, Debbie Marriott, Indy Sandaradura, Simon Tiberi, Vitali Sintchencko, and Charles A Peloquin have no conflicts of interest that are directly relevant to the content of this article.

Ethics approval Not applicable.

Consent to participate Not applicable.

Consent for publication Not applicable.

Availability of data and material Not applicable.

Code availability Not applicable.

Author contributions All authors contributed to the drafting of the manuscript and critically reviewed the final version of the manuscript.

Open Access This article is licensed under a Creative Commons Attribution-NonCommercial 4.0 International License, which permits any non-commercial use, sharing, adaptation, distribution and reproduction in any medium or format, as long as you give appropriate credit to the original author(s) and the source, provide a link to the Creative Commons licence, and indicate if changes were made. The images or other third party material in this article are included in the article's Creative Commons licence, unless indicated otherwise in a credit line to the 
material. If material is not included in the article's Creative Commons licence and your intended use is not permitted by statutory regulation or exceeds the permitted use, you will need to obtain permission directly from the copyright holder. To view a copy of this licence, visit http://creativecommons.org/licenses/by-nc/4.0/.

\section{References}

1. Cowman S, van Ingen J, Griffith DE, Loebinger MR. Nontuberculous mycobacterial pulmonary disease. Eur Respir J. 2019;54:190025.

2. Prevots DR, Marras TK. Epidemiology of human pulmonary infection with nontuberculous mycobacteria: a review. Clin Chest Med. 2015;36:13-34.

3. Haworth CS, Banks J, Capstick T, Fisher AJ, Gorsuch T, Laurenson IF, et al. British Thoracic Society guideline for the management of non-tuberculous mycobacterial pulmonary disease (NTM-PD). BMJ Open Respir Res. 2017;4:e000242.

4. Daley CL, Iaccarino JM, Lange C, Cambau E, Wallace RJJ, Andrejak C, et al. Treatment of nontuberculous mycobacterial pulmonary disease: an official ATS/ERS/ESCMID/IDSA clinical practice guideline. Eur Respir J. 2020;56:2000535.

5. Honda JR, Knight V, Chan ED. Pathogenesis and risk factors for nontuberculous mycobacterial lung disease. Clin Chest Med. 2015;36:1-11.

6. Griffith DE, Aksamit T, Brown-Elliott BA, Catanzaro A, Daley C, Gordin F, et al. An official ATS/IDSA statement: diagnosis, treatment, and prevention of nontuberculous mycobacterial diseases. Am J Respir Crit Care Med. 2007;175:367-416.

7. Hoefsloot W, van Ingen J, Andrejak C, Angeby K, Bauriaud $\mathrm{R}$, Bemer $\mathrm{P}$, et al. The geographic diversity of nontuberculous mycobacteria isolated from pulmonary samples: an NTM-NET collaborative study. Eur Respir J. 2013;42:1604-13.

8. Egelund EF, Fennelly KP, Peloquin CA. Medications and monitoring in nontuberculous mycobacteria infections. Clin Chest Med. 2015;36:55-66.

9. Mouton JW, Muller AE, Canton R, Giske CG, Kahlmeter G, Turnidge J. MIC-based dose adjustment: facts and fables. J Antimicrob Chemother. 2018;73:564-8.

10. Clinical and Laboratory Standard Institute. Susceptibility testing of Mycobacteria, Nocardia spp., and other aerobic actinomycetes. 3rd ed. Approved standard M24-A2. Wayne: Clinical and Laboratory Standards Institute; 2018.

11. Broda A, Jebbari H, Beaton K, Mitchell S, Drobniewski F. Comparative drug resistance of Mycobacterium abscessus and M. chelonae isolates from patients with and without cystic fibrosis in the United Kingdom. J Clin Microbiol. 2013;51:217-23.

12. Brown-Elliott BA, Woods GL. Antimycobacterial susceptibility testing of nontuberculous mycobacteria. J Clin Microbiol. 2019;57:e00834-e919.

13. Schoutrop ELM, Brouwer MAE, Jenniskens JCA, Ferro BE, Mouton JW, Aarnoutse RE, et al. The stability of antimycobacterial drugs in media used for drug susceptibility testing. Diagn Microbiol Infect Dis. 2018;92:305-8.

14. Ceyssens P-J, Soetaert K, Timke M, Van den Bossche A, Sparbier $\mathrm{K}$, De Cremer K, et al. Matrix-assisted laser desorption ionization-time of flight mass spectrometry for combined species identification and drug sensitivity testing in mycobacteria. J Clin Microbiol. 2017;55:624-34.

15. Huang Y-C, Liu M-F, Shen G-H, Lin C-F, Kao C-C, Liu P-Y, Shi Z-Y. Clinical outcome of Mycobacterium abscessus infection and antimicrobial susceptibility testing. J Microbiol Immunol Infect. 2010;43:401-6.

16. Li G, Pang H, Guo Q, Huang M, Tan Y, Li C, et al. Antimicrobial susceptibility and MIC distribution of 41 drugs against clinical isolates from China and reference strains of nontuberculous mycobacteria. Int J Antimicrob Agents. 2017;49:364-74.

17. Litvinov V, Makarova M, Galkina K, Khachaturiants E, Krasnova M, Guntupova L, et al. Drug susceptibility testing of slowly growing non-tuberculous mycobacteria using slomyco test-system. PLoS ONE. 2018;13:e0203108.

18. van Ingen J, Totten SE, Helstrom NK, Heifets LB, Boeree MJ, Daley CL. In vitro synergy between clofazimine and amikacin in treatment of nontuberculous mycobacterial disease. Antimicrob Agents Chemother. 2012;56:6324-7.

19. Brown-Elliott BA, Wallace RJ Jr. In vitro susceptibility testing of tedizolid against nontuberculous Mycobacteria. J Clin Microbiol. 2017;55:1747-54.

20. Huh HJ, Kim S-Y, Jhun BW, Shin SJ, Koh W-J. Recent advances in molecular diagnostics and understanding mechanisms of drug resistance in nontuberculous mycobacterial diseases. Infect Genet Evol. 2019;72:169-82.

21. Kim HY, Sintchenko V, Alffenaar JW. Nontuberculosis mycobacteria infections: would there be pharmacodynamics without pharmacokinetics? Eur Respir J. 2019;54:1901508.

22. Mougari F, Loiseau J, Veziris N, Bernard C, Bercot B, Sougakoff $\mathrm{W}$, et al. Evaluation of the new GenoType NTM-DR kit for the molecular detection of antimicrobial resistance in non-tuberculous mycobacteria. J Antimicrob Chemother. 2017;72:1669-77.

23. Srivastava S, Pasipanodya JG, Meek C, Leff R, Gumbo T. Multidrug-resistant tuberculosis not due to noncompliance but to between-patient pharmacokinetic variability. J Infect Dis. 2011;204:1951-9.

24. Gumbo T, Angulo-Barturen I, Ferrer-Bazaga S. Pharmacokinetic-pharmacodynamic and dose-response relationships of antituberculosis drugs: recommendations and standards for industry and academia. J Infect Dis. 2015;211(Suppl.):S96-106.

25. Mouton JW, Vinks AA. Pharmacokinetic/pharmacodynamic modelling of antibacterials in vitro and in vivo using bacterial growth and kill kinetics: the minimum inhibitory concentration versus stationary concentration. Clin Pharmacokinet. 2005;44:201-10.

26. Ferro BE, van Ingen J, Wattenberg M, van Soolingen D, Mouton JW. Time-kill kinetics of slowly growing mycobacteria common in pulmonary disease. J Antimicrob Chemother. 2015;70:2838-43.

27. Ferro BE, van Ingen J, Wattenberg M, van Soolingen D, Mouton JW. Time-kill kinetics of antibiotics active against rapidly growing mycobacteria. J Antimicrob Chemother. 2015;70:811-7.

28. Deshpande D, Pasipanodya JG, Gumbo T. Azithromycin dose to maximize efficacy and suppress acquired drug resistance in pulmonary Mycobacterium avium disease. Antimicrob Agents Chemother. 2016;60:2157-63.

29. Deshpande D, Srivastava S, Meek C, Leff R, Gumbo T. Ethambutol optimal clinical dose and susceptibility breakpoint identification by use of a novel pharmacokinetic-pharmacodynamic model of disseminated intracellular Mycobacterium avium. Antimicrob Agents Chemother. 2010;54:1728-33.

30. Ferro BE, Srivastava S, Deshpande D, Sherman CM, Pasipanodya JG, van Soolingen D, et al. Amikacin pharmacokinetics/pharmacodynamics in a novel hollow-fiber Mycobacterium abscessus disease model. Antimicrob Agents Chemother. 2015;60:1242-8.

31. Deshpande D, Srivastava S, Pasipanodya JG, Gumbo T. Linezolid as treatment for pulmonary Mycobacterium avium disease. J Antimicrob Chemother. 2017;72:i24-9. 
32. Srivastava S, Gumbo T. In vitro and in vivo modeling of tuberculosis drugs and its impact on optimization of doses and regimens. Curr Pharm Des. 2011;17:2881-8.

33. Srivastava S, Deshpande D, Gumbo T. Failure of the azithromycin and ethambutol combination regimen in the hollow-fibre system model of pulmonary Mycobacterium avium infection is due to acquired resistance. J Antimicrob Chemother. 2017;72:i20-3.

34. Winthrop K. Comparison of two- versus three-antibiotic therapy for pulmonary Mycobacterium Avium Complex Disease (MAC2v3). ClinicalTrials.gov Identifier: NCT03672630.

35. Chapagain M, Gumbo T, Heysell SK, Srivastava S. Comparison of a novel regimen of rifapentine, tedizolid, and minocycline with standard regimens for treatment of the pulmonary Mycobacterium kansasii. Antimicrob Agents Chemother. 2020;64:e00810-e820.

36. Ferro BE, Srivastava S, Deshpande D, Pasipanodya JG, van Soolingen D, Mouton JW, et al. Failure of the amikacin, cefoxitin, and clarithromycin combination regimen for treating pulmonary Mycobacterium abscessus infection. Antimicrob Agents Chemother. 2016;60:6374-6.

37. Gilljam M, Berning SE, Peloquin CA, Strandvik B, Larsson LO. Therapeutic drug monitoring in patients with cystic fibrosis and mycobacterial disease. Eur Respir J. 1999;14:347-51.

38. van Ingen J, Egelund EF, Levin A, Totten SE, Boeree MJ, Mouton JW, et al. The pharmacokinetics and pharmacodynamics of pulmonary Mycobacterium avium complex disease treatment. Am J Respir Crit Care Med. 2012;186:559-65.

39. Bolhuis MS, van Altena R, van Soolingen D, de Lange WCM, Uges DRA, van der Werf TS, et al. Clarithromycin increases linezolid exposure in multidrug-resistant tuberculosis patients. Eur Respir J. 2013;42:1614-21.

40. Ashizawa N, Tsuji Y, Kawago K, Higashi Y, Tashiro M, Nogami $\mathrm{M}$, et al. Successful treatment of methicillin-resistant Staphylococcus aureus osteomyelitis with combination therapy using linezolid and rifampicin under therapeutic drug monitoring. J Infect Chemother. 2016;22:331-4.

41. Zuckerman JM, Qamar F, Bono BR. Review of macrolides (azithromycin, clarithromycin), ketolids (telithromycin) and glycylcyclines (tigecycline). Med Clin N Am. 2011;95:761-91, viii.

42. van Altena R, Dijkstra JA, van der Meer ME, Borjas Howard JF, Kosterink JGW, van Soolingen D, et al. Reduced chance of hearing loss associated with therapeutic drug monitoring of aminoglycosides in the treatment of multidrug-resistant tuberculosis. Antimicrob Agents Chemother. 2017;61:e01400-e1416.

43. Alffenaar J-WC, van Altena R, Harmelink IM, Filguera P, Molenaar E, Wessels AMA, et al. Comparison of the pharmacokinetics of two dosage regimens of linezolid in multidrug-resistant and extensively drug-resistant tuberculosis patients. Clin Pharmacokinet. 2010;49:559-65.

44. Abdul Aziz MH, Alffenaar JWC, Bassetti M, Bracht H, Dimopoulos G, Marriott D, et al. Antimicrobial therapeutic drug monitoring in critically ill adult patients: a Position Paper. Intensive Care Med. 2020;46:1127-53.

45. Heysell SK, Moore JL, Keller SJ, Houpt ER. Therapeutic drug monitoring for slow response to tuberculosis treatment in a state control program, Virginia, USA. Emerg Infect Dis. 2010;16:1546-53.

46. Alkabab Y, Keller S, Dodge D, Houpt E, Staley D, Heysell S. Early interventions for diabetes related tuberculosis associate with hastened sputum microbiological clearance in Virginia, USA. BMC Infect Dis. 2017;17:125.

47. van den Elsen SHJ, Sturkenboom MGG, Akkerman OW, Manika K, Kioumis IP, van der Werf TS, et al. Limited sampling strategies using linear regression and the Bayesian approach for therapeutic drug monitoring of moxifloxacin in tuberculosis patients. Antimicrob Agents Chemother. 2019;63:e00384-e419.

48. Dijkstra JA, van Altena R, Akkerman OW, de Lange WCM, Proost JH, van der Werf TS, et al. Limited sampling strategies for therapeutic drug monitoring of amikacin and kanamycin in patients with multidrug-resistant tuberculosis. Int J Antimicrob Agents. 2015;46:332-7.

49. Ferro BE, Srivastava S, Deshpande D, Pasipanodya JG, van Soolingen D, Mouton JW, et al. Moxifloxacin's limited efficacy in the hollow-fiber model of Mycobacterium abscessus disease. Antimicrob Agents Chemother. 2016;60:3779-85.

50. Wehrli W. Rifampin: mechanisms of action and resistance. Rev Infect Dis. 1983;5(Suppl. 3):S407-11.

51. Haworth CS, Banks J, Capstick T, Fisher AJ, Gorsuch T, Laurenson IF, et al. British Thoracic Society guidelines for the management of non-tuberculous mycobacterial pulmonary disease (NTM-PD). Thorax. 2017;72:ii1-64.

52. Aziz DB, Low JL, Wu M-L, Gengenbacher M, Teo JWP, Dartois $\mathrm{V}$, et al. Rifabutin is active against Mycobacterium abscessus complex. Antimicrob Agents Chemother. 2017;61:e00155-e217.

53. Ganapathy US, Dartois V, Dick T. Repositioning rifamycins for Mycobacterium abscessus lung disease. Expert Opin Drug Discov. 2019;14:867-78.

54. Woods GL, Brown-Elliott BA, Conville PS, Desmond EP, Hall GS, Lin G, et al. 2nd ed. Wayne (PA): Clinical and Laboratory Standards Institute; 2011. Report No.: M24-A2.

55. Magis-Escurra C, Alffenaar JW, Hoefnagels I, Dekhuijzen PNR, Boeree MJ, van Ingen J, et al. Pharmacokinetic studies in patients with nontuberculous mycobacterial lung infections. Int J Antimicrob Agents. 2013;42:256-61.

56. Koh W-J, Jeong B-H, Jeon K, Lee S-Y, Shin SJ. Therapeutic drug monitoring in the treatment of Mycobacterium avium complex lung disease. Am J Respir Crit Care Med. 2012;186:797-802.

57. Stott KE, Pertinez H, Sturkenboom MGG, Boeree MJ, Aarnoutse $\mathrm{R}$, Ramachandran $\mathrm{G}$, et al. Pharmacokinetics of rifampicin in adult TB patients and healthy volunteers: a systematic review and meta-analysis. J Antimicrob Chemother. 2018;73:2305-13.

58. Satyaraddi A, Velpandian T, Sharma SK, Vishnubhatla S, Sharma A, Sirohiwal A, et al. Correlation of plasma anti-tuberculosis drug levels with subsequent development of hepatotoxicity. Int J Tuberc Lung Dis. 2014;18(188-95):i-iii.

59. Dinos GP. The macrolide antibiotic renaissance. Br J Pharmacol. 2017;174:2967-83.

60. Moon SM, Park HY, Kim S-Y, Jhun BW, Lee H, Jeon K, et al. Clinical characteristics, treatment outcomes, and resistance mutations associated with macrolide-resistant Mycobacterium avium complex lung disease. Antimicrob Agents Chemother. 2016;60:6758-65.

61. Morimoto K, Namkoong H, Hasegawa N, Nakagawa T, Morino E, Shiraishi Y, et al. Macrolide-resistant Mycobacterium avium complex lung disease: analysis of 102 consecutive cases. Ann Am Thorac Soc. 2016;13:1904-11.

62. Brown-Elliott BA, Nash KA, Wallace RJJ. Antimicrobial susceptibility testing, drug resistance mechanisms, and therapy of infections with nontuberculous mycobacteria. Clin Microbiol Rev. 2012;25:545-82.

63. Lalak NJ, Morris DL. Azithromycin clinical pharmacokinetics. Clin Pharmacokinet. 1993;25:370-4.

64. Rodvold KA. Clinical pharmacokinetics of clarithromycin. Clin Pharmacokinet. 1999;37:385-98.

65. Gumbo T, Louie A, Deziel MR, Parsons LM, Salfinger M, Drusano GL. Selection of a moxifloxacin dose that suppresses drug resistance in Mycobacterium tuberculosis, by use of an in vitro pharmacodynamic infection model and mathematical modeling. J Infect Dis. 2004;190:1642-51. 
66. Deshpande D, Pasipanodya JG, Mpagama SG, Bendet P, Srivastava S, Koeuth T, et al. Levofloxacin pharmacokinetics/pharmacodynamics, dosing, susceptibility breakpoints, and artificial intelligence in the treatment of multidrug-resistant tuberculosis. Clin Infect Dis. 2018;67:S293-302.

67. Cho EH, Huh HJ, Song DJ, Moon SM, Lee S-H, Shin SY, et al. Differences in drug susceptibility pattern between Mycobacterium avium and Mycobacterium intracellulare isolated in respiratory specimens. J Infect Chemother. 2018;24:315-8.

68. Basille D, Jounieaux V, Andréjak C. Treatment of other nontuberculous mycobacteria. Semin Respir Crit Care Med. 2018;39:377-82.

69. Shen Y, Wang X, Jin J, Wu J, Zhang X, Chen J, et al. In vitro susceptibility of Mycobacterium abscessus and Mycobacterium fortuitum isolates to 30 antibiotics. Biomed Res Int. 2018;2018:4902941.

70. Ryu YJ, Koh W-J, Daley CL. Diagnosis and treatment of nontuberculous mycobacterial lung disease: clinicians' perspectives. Tuberc Respir Dis (Seoul). 2016;79:74-84.

71. Deshpande D, Srivastava S, Meek C, Leff R, Hall GS, Gumbo T. Moxifloxacin pharmacokinetics/pharmacodynamics and optimal dose and susceptibility breakpoint identification for treatment of disseminated Mycobacterium avium infection. Antimicrob Agents Chemother. 2010;54:2534-9.

72. Srivastava S, Pasipanodya J, Sherman CM, Meek C, Leff R, Gumbo T. Rapid drug tolerance and dramatic sterilizing effect of moxifloxacin monotherapy in a novel hollow-fiber model of intracellular Mycobacterium kansasii disease. Antimicrob Agents Chemother. 2015;59:2273-9.

73. Nix DEDE, Adam RDRD, Auclair B, Krueger TSTS, Godo PGPG, Peloquin CACA. Pharmacokinetics and relative bioavailability of clofazimine in relation to food, orange juice and antacid. Tuberculosis (Edinb). 2004;84:365-73.

74. Kwak N, Whang J, Yang JS, Kim TS, Kim SA, Yim J-J. Minimal inhibitory concentration of clofazimine among clinical isolates of nontuberculous mycobacteria and its impact on treatment outcome. Chest. 2021;159(2):517-23.

75. van Ingen $J$, van der Laan $T$, Dekhuijzen $R$, Boeree $M$, van Soolingen D. In vitro drug susceptibility of 2275 clinical nontuberculous Mycobacterium isolates of 49 species in the Netherlands. Int J Antimicrob Agents. 2010;35:169-73.

76. Ruth MM, Sangen JJN, Remmers K, Pennings LJ, Svensson E, Aarnoutse RE, et al. A bedaquiline/clofazimine combination regimen might add activity to the treatment of clinically relevant non-tuberculous mycobacteria. J Antimicrob Chemother. 2019;74:935-43.

77. Srivastava S, Gumbo T. Clofazimine for the treatment of Mycobacterium kansasii. Antimicrob Agents Chemother. 2018;62:e00248-e318.

78. Banaschewski B, Verma D, Pennings LJ, Zimmerman M, Ye Q, Gadawa J, et al. Clofazimine inhalation suspension for the aerosol treatment of pulmonary nontuberculous mycobacterial infections. J Cyst Fibros. 2019;18:714-20.

79. van Ingen J, Ferro BE, Hoefsloot W, Boeree MJ, van Soolingen D. Drug treatment of pulmonary nontuberculous mycobacterial disease in HIV-negative patients: the evidence. Expert Rev Anti Infect Ther. 2013;11:1065-77.

80. Jarand J, Davis JP, Cowie RL, Field SK, Fisher DA. Long-term follow-up of Mycobacterium avium complex lung disease in patients treated with regimens including clofazimine and/or rifampin. Chest. 2016;149:1285-93.

81. Carey GB, Tebas P, Vinnard C, Kim D, Hadjiliadis D, HansenFlaschen J, et al. Clinical outcomes of clofazimine use for rapidly growing mycobacterial infections. Open Forum Infect Dis. 2019;6:1-9.
82. Abdelwahab MT, Wasserman S, Brust JCM, Gandhi NR, Meintjes G, Everitt D, et al. Clofazimine pharmacokinetics in patients with TB: dosing implications. J Antimicrob Chemother. 2020;75:3269-77.

83. Peloquin CA, Namdar R, Dodge AA, Nix DE. Pharmacokinetics of isoniazid under fasting conditions, with food, and with antacids. Int J Tuberc Lung Dis. 1999;3:703-10.

84. Floto RA, Olivier KN, Saiman L, Daley CL, Herrmann J-L, Nick JA, et al. US Cystic Fibrosis Foundation and European Cystic Fibrosis Society consensus recommendations for the management of non-tuberculous mycobacteria in individuals with cystic fibrosis. Thorax. 2016;71(Suppl. 1):i1-22.

85. Shulha JA, Escalante P, Wilson JW. Pharmacotherapy approaches in nontuberculous mycobacteria infections. Mayo Clin Proc. 2019;94:1567-81.

86. Sturkenboom MGG, Simbar N, Akkerman OW, Ghimire S, Bolhuis MS, Alffenaar J-WC. Amikacin dosing for MDR tuberculosis: a systematic review to establish or revise the current recommended dose for tuberculosis treatment. Clin Infect Dis. 2018;67:S303-7.

87. Peloquin CA, Berning SE, Nitta AT, Simone PM, Goble M, Huitt GA, et al. Aminoglycoside toxicity: daily versus thrice-weekly dosing for treatment of mycobacterial diseases. Clin Infect Dis. 2004;38:1538-44.

88. Muñoz-Egea M-C, Carrasco-Antón N, Esteban J. State-of-the-art treatment strategies for nontuberculous mycobacteria infections. Expert Opin Pharmacother. 2020;21:969-81.

89. Wallace RJJ, Brown-Elliott BA, Crist CJ, Mann L, Wilson RW. Comparison of the in vitro activity of the glycylcycline tigecycline (formerly GAR-936) with those of tetracycline, minocycline, and doxycycline against isolates of nontuberculous mycobacteria. Antimicrob Agents Chemother. 2002;46:3164-7.

90. Conte JE, Golden JA, Kelly MG, Zurlinden E. Steady-state serum and intrapulmonary pharmacokinetics and pharmacodynamics of tigecycline. Int J Antimicrob Agents. 2005;25:523-9.

91. Ferro BE, Srivastava S, Deshpande D, Pasipanodya JG, van Soolingen D, Mouton JW, et al. Tigecycline is highly efficacious against Mycobacterium abscessus pulmonary disease. Antimicrob Agents Chemother. 2016;60:2895-900.

92. Opal S, File TM, van der Poll T, Tzanis E, Chitra S, McGovern PC. An integrated safety summary of omadacycline, a novel aminomethylcycline antibiotic. Clin Infect Dis. 2019;69:S40-7.

93. Gumbo T, Cirrincione K, Srivastava S. Repurposing drugs for treatment of Mycobacterium abscessus: a view to a kill. J Antimicrob Chemother. 2020;75:1212-7.

94. European Medicines Agency. Nuzyra: withdrawal of the marketing authorisation application. Available from: https://www.ema. europa.eu/en/medicines/human/withdrawn-applications/nuzyra. Accessed 15 Feb 2021.

95. Yuste JR, Bertó J, Del Pozo JL, Leiva J. Prolonged use of tedizolid in a pulmonary non-tuberculous mycobacterial infection after linezolid-induced toxicity. J Antimicrob Chemother. 2017;72:625-8.

96. Mok S, Hannan MM, Nölke L, Stapleton P, O'Sullivan N, Murphy $\mathrm{P}$, et al. Antimicrobial susceptibility of clinical and environmental Mycobacterium chimaera isolates. Antimicrob Agents Chemother. 2019;63(9):e00755-e819.

97. Prokocimer P, Bien P, Surber J, Mehra P, DeAnda C, Bulitta $\mathrm{JB}$, et al. Phase 2, randomized, double-blind, dose-ranging study evaluating the safety, tolerability, population pharmacokinetics, and efficacy of oral torezolid phosphate in patients with complicated skin and skin structure infections. Antimicrob Agents Chemother. 2011;55:583-92.

98. Deshpande D, Srivastava S, Pasipanodya JG, Lee PS, Gumbo T. Tedizolid is highly bactericidal in the treatment of pulmonary 
Mycobacterium avium complex disease. J Antimicrob Chemother. 2017;72:i30-5.

99. Dubée V, Bernut A, Cortes M, Lesne T, Dorchene D, Lefebvre A-L, et al. $\beta$-Lactamase inhibition by avibactam in Mycobacterium abscessus. J Antimicrob Chemother. 2015;70:1051-8.

100. Kaushik A, Gupta C, Fisher S, Story-Roller E, Galanis C, Parrish $\mathrm{N}$, et al. Combinations of avibactam and carbapenems exhibit enhanced potencies against drug-resistant Mycobacterium abscessus. Future Microbiol. 2017;12:473-80.

101. Deshpande D, Srivastava S, Chapagain ML, Lee PS, Cirrincione $\mathrm{KN}$, Pasipanodya JG, et al. The discovery of ceftazidime/avibactam as an anti-Mycobacterium avium agent. J Antimicrob Chemother. 2017;72:i36-42.

102. Rominski A, Schulthess B, Müller DM, Keller PM, Sander P. Effect of $\beta$-lactamase production and $\beta$-lactam instability on MIC testing results for Mycobacterium abscessus. J Antimicrob Chemother. 2017;72:3070-8.

103. Balemans W, Vranckx L, Lounis N, Pop O, Guillemont J, Vergauwen $\mathrm{K}$, et al. Novel antibiotics targeting respiratory ATP synthesis in Gram-positive pathogenic bacteria. Antimicrob Agents Chemother. 2012;56:4131-9.

104. Pang Y, Zheng H, Tan Y, Song Y, Zhao Y. In vitro activity of bedaquiline against nontuberculous mycobacteria in China. Antimicrob Agents Chemother. 2017;61:e02627-e2716.

105. Ji B, Lefrançois S, Robert J, Chauffour A, Truffot C, Jarlier V. In vitro and in vivo activities of rifampin, streptomycin, amikacin, moxifloxacin, R207910, linezolid, and PA-824 against Mycobacterium ulcerans. Antimicrob Agents Chemother. 2006;50:1921-6.

106. Philley JV, Wallace RJJ, Benwill JL, Taskar V, Brown-Elliott BA, Thakkar F, et al. Preliminary results of bedaquiline as salvage therapy for patients with nontuberculous mycobacterial lung disease. Chest. 2015;148:499-506.

107. Aguilar-Ayala DA, Cnockaert M, André E, Andries K, Gonzalez-Y-Merchand JA, Vandamme P, et al. In vitro activity of bedaquiline against rapidly growing nontuberculous mycobacteria. J Med Microbiol. 2017;66:1140-3.

108. Lounis N, Gevers T, Van den Berg J, Vranckx L, Andries K. ATP synthase inhibition of Mycobacterium avium is not bactericidal. Antimicrob Agents Chemother. 2009;53:4927-9.

109. Alexander DC, Vasireddy R, Vasireddy S, Philley JV, BrownElliott BA, Perry BJ, et al. Emergence of mmpT5 variants during bedaquiline treatment of Mycobacterium intracellulare lung disease. J Clin Microbiol. 2017;55:574-84.

110. Doi N, Disratthakit A. Characteristic antimycobacterial spectra of the novel anti-TB drug candidates OPC-67683 and PA-824 [poster F1-F1377a]. Interscience Conference on Antimicrobial Agents and Chemotherapy. San Francisco, 27-30 September 2006, 46th conference.

111. Märtson A-G, Sturkenboom MGG, Stojanova J, Cattaneo D, Hope W, Marriott D, et al. How to design a study to evaluate therapeutic drug monitoring in infectious diseases? Clin Microbiol Infect. 2020;26:1008-16.

112. Timmins GS. What are the challenges in commercial non-tuberculous mycobacteria (NTM) drug discovery and how should we move forward? Expert Opin Drug Discov. 2020;15(1):7-9.

113. Park SC, Kang MJ, Han CH, Lee SM, Kim CJ, Lee JM, Kang YA. Prevalence, incidence, and mortality of nontuberculous mycobacterial infection in Korea: a nationwide population-based study. BMC Pulm Med. 2019;19:140.

114. Winthrop KL, Marras TK, Adjemian J, Zhang H, Wang P, Zhang Q. Incidence and prevalence of nontuberculous mycobacterial lung disease in a large U.S. managed care health plan, 20082015. Ann Am Thorac Soc. 2020;17:178-85.

115. Wheeler C, Berkley S. Initial lessons from public-private partnerships in drug and vaccine development. Bull World Health Organ. 2001;79:728-34. 